\title{
Galactic starburst NGC 3603 from X-rays to radio
}

\author{
Anthony F.J. Moffat \\ Département de Physique, Université de Montréal, \\ C.P. 6128, Succ. Centre-Ville, Montréal, QC H3C 3J7, Canada, and \\ Observatoire du mont Mégantic, Canada
}

and the XMEGA consortium

\begin{abstract}
While NGC 3603 is often quoted as the most massive visible Giant H II Region in the Galaxy, there are other similar and even more massive regions now being found towards the inner Galaxy in the near-IR. Nevertheless, NGC 3603 still retains the status of clone to the dense core-object in 30Dor, R 136 - but $7 \times$ closer and $49 \times$ less crowded! This paper summarizes the most recent findings concerning NGC 3603's color-magnitude diagram (CMD), initial mass function (IMF), mass segregation and stellar content - including its unusually luminous H-rich WNL members - down to its pre-main-sequence stars near the H-burning limit. Of special relevance are new high-resolution X-ray and radio images as related to merging/colliding winds and three massive proplydlike objects. NGC 3603 is a somewhat younger, hotter, scaled-down version of typical starbursts found in other galaxies.
\end{abstract}

\section{NGC 3603: vital statistics}

NGC 3603 is a Galactic Giant H II Region located in the far part of the Carina arm at a distance of $7 \mathrm{kpc}$ (galactocentric distance $R=8.5 \mathrm{kpc}$, for $\mathrm{R}_{\odot}=8.0 \mathrm{kpc}$ ) at $l=291.6^{\circ}, b=-0.5^{\circ}$. (It is useful to note that for this distance $1^{\prime}=2 \mathrm{pc}$.) The $\mathrm{H}$ II region has a core-halo structure, with radii of 3.5 and $25 \mathrm{pc}$, respectively. The metallicity is slightly sub-solar, like Orion. Its most massive stars are found out to $r \simeq 2 \mathrm{pc}$, compared to $r \simeq 5 \mathrm{pc}$ for its faintest pre-main-sequence stars. Corresponding King core radii are $\sim 0.1 \mathrm{pc}$ and $\sim 1 \mathrm{pc}$, respectively. Obscured behind $A_{V}=4.6 \mathrm{mag}$ of normal IS extinction (reaching up to $\sim 6 \mathrm{mag}$ for member stars North and South of the main cluster), NGC 3603 contains some $5000 \mathrm{M}_{\odot}$ in stars, $\sim 50 \mathrm{M}_{\odot}$ of which are of type $\mathrm{O}$, many of the hottest types known, and three central H-rich WNLh+abs stars. Comprising about $30 \%$ of the H II mass, the stars reach a central density of $\sim 10^{5} \mathrm{M}_{\odot} \mathrm{pc}^{-3}$. The total luminosity is $\sim 10^{7} \mathrm{~L}_{\odot}$ and the age is nearly coeval close to its core at $\sim 1 \mathrm{Myr}$, reaching some $10 \mathrm{Myr}$ to the North and less than $1 \mathrm{Myr}$ for heavily obscured, massive protostars towards the South/South-West. A central stellar bubble with radius $r \simeq 0.6 \mathrm{pc}$ has been evacuated by the combined strong winds of the central cluster, where $\mathrm{O} / \mathrm{WR}$ stars dominate. A previous recent review of NGC 3603 was given by 
Drissen (1999). A more elaborate version of this paper is given by Moffat et al. (2002).

\section{NGC 3603: its place among peers}

NGC 3603 is often quoted as 'the most massive visible giant $\mathrm{HII}$ (GH II) region in the Galaxy'. However, other Galactic GH II regions are now known to rival or even surpass NGC 3603, although these are mostly only seen in the near-IR towards the inner Galaxy (e.g., the Central Cluster, Arches Cluster, Quintuplet Cluster, W 42, W 49, W 51, W 53, ...). In the rest of the Local Group, where resolution is still adequate to resolve individual stars, clearly 30 Dor is the most massive GH II region, surpassing NGC 3603 by over an order of magnitude. Nevertheless, 30 Dor's central dense core, R 136, and NGC 3603, are clones when it comes to massive stars within $1 \mathrm{pc}$ of their centres (Moffat et al. 1994). Remarkably, both objects have three central WR stars that dominate the core light. In actual fact, NGC 3603 is even more peaked in its centre than R 136 . Crowther and Dessart (1998) give an inventory of the massive stars in both objects: restricting to $r \leq 1 \mathrm{pc}$ and $\mathrm{M}_{V} \leq-4.5$, one finds in NGC 360339 stars, of which 25 are of type O3-4 and three of type WN6h+abs, HD 97950-A1, -B, and $-\mathrm{C}$, listed as WR 43a, WR 43b and WR 43c by van der Hucht (2001); and in R 13634 stars, of which 25 of type O3-4 and four WN5h, listed as BAT99-106, BAT99-108, BAT99-109 and BAT99-110 by Breysacher et al. (1999). Despite these similarities, the fact remains that NGC 3603 is $7 \times$ closer than R 136 and thus $49 \times$ less crowded! For the same absolute magnitude in a given band, $\mathrm{M}_{\lambda}$, stars in both clusters are about equally bright in the visual, while at near-IR wavelengths, NGC 3603's stars are considerably brighter (because of less $1 / d^{2}$ dilution), while at UV wavelengths, the reverse is true (R 136 is less extincted). NGC 3603's stars are also easier to observe in (non-soft) X-rays because of the distance factor, which also favours the resolution.

\section{Stellar content}

This is best studied at visible and near-IR wavelengths in NGC 3603.

\subsection{Photometry}

The latest, most complete optical photometric study is that of Grebel et al. (2003) in broadband $V R I$ and narrowband $\mathrm{H} \alpha$ using mainly HST-wFPC2. Of particular interest in this study, after establishing that the interstellar extinction law is normal, is the comparison of the color-magnitude diagrams (CMDs) on the WFPC2 images in each case of NGC 3603 compared to R 136 and its surroundings (from Hunter et al. 1995). As one progesses down the CMD of NGC 3603, its main-sequence ends where the pre main-sequence stars begin, implying that NGC 3603 is essentially co-eval with an age close to 1 Myr. The R 136 cluster on the other hand has an extended region with filled-in transition from the mainsequence to the pre main-sequence, implying an age spread of $1-5 \mathrm{Myr}$, with the massive stars having formed most recently. However, it should be noted that the WFPC 2 field includes $7^{2}=49 \times$ larger area in parsecs centered on R 136 than in NGC 3603, thus explaining why the R 136 cluster contains many more stars but may be less co-eval. 
Grebel et al. (2003) have also looked at emission-line stars using the narrowband $\mathrm{H} \alpha$ vs. $R$-filter images. They find few $\mathrm{H} \alpha$ emitters among low-mass, pre main-sequence stars ( $\grave{a}$ la T Tauri). This may signify rapid destruction of protostellar disks in the strong radiation field of the hot stars of NGC 3603. It may also lead to supression of planet formation!

As for the IMF, including all stars down to 1-2 $\mathrm{M}_{\odot}$, Grebel et al. (2003) find a flat IMF in the core of NGC $3603(\Gamma=0)$, with slope increasing gradually with radius up to a Salpeter value $(\Gamma=1.35)$ beyond some $0.6 \mathrm{pc}$. This implies strong mass segregation, suggesting that the intense UV radiation of high-mass stars suppresses the formation of low-mass stars. This situation is similar to that found in R 136 (Hunter et al. 1995, 1996; Selman et al. 1999).

Grebel et al. (2003) note a trend in relaxation times ranging from $<1 \mathrm{Myr}$ in the dense clusters near the Galactic Centre like Arches, through $\sim 2 \mathrm{Myr}$ for NGC 3603 to $\sim 40 \mathrm{Myr}$ for the R 136 cluster. From these, evaporation times of $\leq 5,<240$ and $\sim 5000 \mathrm{Myr}$ are deduced, respectively. This would then imply that clusters near the Galactic Centre evaporate fast (self-destruct via internal dynamics), NGC 3603 medium fast (since $t_{\text {rel }} \propto$ age), while the R 136 cluster will evaporate so slowly that it could become a populous cluster later, of the kind seen elsewhere in the LMC. However, one wonders that, if one were to separate out R 136 from its surroundings (like NGC 3603), would this not change the result?

Brandl et al. (1999) have obtained deep JHK images of NGC 3603 using one of the VLT $8 \mathrm{~m}$ telescopes. This offers the best way to probe the pre-mainsequence (PMS) population. They find within a radius of $\sim 1 \mathrm{pc}$ from the centre, a PMS of age $0.3-1.0 \mathrm{Myr}$ down essentially to the limit of H-burning $\left(0.1 \mathrm{M}_{\odot}\right)$. A detailed IMF from these data is pending, although it is already clear that low-mass stars can form in the presence of massive stars in massive starbursts!

Nürnberger \& Petr-Gotzens (2002) have obtained very deep, wide-field $K_{\mathrm{s}}$ band images of NGC 3603, also at the VLT. They find a maximum extent of $r \simeq 5 \mathrm{pc}$ for the faintest stars, which is a factor 2.5 greater than that for the most massive stars. Despite the lack of dynamical equilibrium and the clear, but not severe, elongation of the cluster's fainter stars in the Galactic plane (thus either tidal or in the form of the original molecular cloud), King models do appear to fit the data fairly well. These lead to core radii varying from $\sim 0.1 \mathrm{pc}$ for the brightest $(\mathrm{OB})$ stars, through $\sim 0.25 \mathrm{pc}$ for intermediate-mass stars, to $\sim 1.2 \mathrm{pc}$ for the faintest PMS stars. Again, this implies that the massive stars are far more concentrated to the centre than are low-mass stars. This is similar to R 136, in which Brandl et al. (1996) suggest that this is the result of the initial conditions or violent relaxation. Given these results, it does seem strange that the global IMF can even become Salpeter-like beyond $r \simeq 0.6 \mathrm{pc}$, as suggested for NGC 3603 by Grebel et al. (2003) or for R 136 by Selman et al. (1999) and Hunter et al. (1995, 1996).

\subsection{Spectroscopy}

Given the increased degeneracy as one goes to the most massive stars, spectroscopy is essential for an accurate assessment of the massive-star content of NGC 3603. The first set of quality optical spectra was obtained by Moffat (1983) for 13 member stars outside the dense core, followed by HST-FOS spectra for 14 
stars in the dense core (Drissen et al. 1995). A more complete study, including radial velocities is under way by Skalkowski et al. (in preparation).

The results can be summarized as follows. NGC 3603 contains at least 50 $\mathrm{O} / \mathrm{WR}$ stars, all of which are essentially co-eval, except for 2-3 OB supergiants outside the central dense core. Its WR stars WR 43a, WR 43b and WR 43c are all of type WN6h+abs in the system of Smith et al. (1996). A spectral montage of the brightest stars in the dense core shows a remarkably continuous sequence in wind strength, ranging from star WR 43b down through WR 43a, WR 43c and into the $\mathrm{O} 3$ supergiants and main-sequence O3 stars. NGC 3603's known $\mathrm{OB} / \mathrm{WR}$ stars can easily account for the ionizing photons required to produce the surrounding giant $\mathrm{H}$ II region. However, the central wind bubble is much too small for such a large collection of hot, luminous stars, unless the age (or duration of high mass-loss rates) is vastly overestimated.

Quantitative analysis was carried out on the optical spectra of the three WR stars in NGC 3603 by Crowther \& Dessart (1998) and Schmutz \& Drissen (1999). These two studies generally agree on the following mean characteristics of each of these three stars: $\mathrm{H} / \mathrm{He} \simeq 3-6$ by number (compared to $\leq 2.5$ for classical WR stars), $L \simeq 10^{6.2} \mathrm{~L}_{\odot}, \dot{M} \simeq 5 \times 10^{-5} \mathrm{M}_{\odot} \mathrm{yr}^{-1}$ and $M_{\text {init }} \geq 100 \mathrm{M}_{\odot}$. These do not correspond to the parameters of classical WR stars, suggesting that these stars are probably extremely massive and luminous main-sequence stars that can drive strong, hot winds, as suggested by de Koter et al. (1998) for the central WR stars in R 136. Thus, stars WR 43a, WR 43b and WR 43c in NGC 3603 are also similar to WR 25 in the Carina Nebula and to R 144, often considered to be the most luminous and massive star in 30 Dor.

Various projects are being carried out in an attempt to obtain the masses of the most massive stars. Recently, Massey et al. (2002) published HST spectral work on four O3 binaries in the R 136 cluster. Skalkowski et al. (in preparation) are looking for binaries in 30 Dor and NGC 3603. Schnurr et al. (in preparation) are looking at the entire WNL population in the LMC. Especially important in this context are the WNLh stars, which may be the most massive. In the case of NGC 3603 and R 136, short-period binary motion of unresolved He II $4686 \AA$ emission was discovered in the cores of these two objects by Moffat \& Niemela (1984) and Moffat \& Seggewiss (1983), confirmed in both cases by Moffat et al. (1985). While one of the WN6h+abs stars in NGC 3603 must be a $3.7720 \mathrm{~d}$ binary, one of the WN5h stars near the centre of R 136 must be a $4.377 \mathrm{~d}$ binary. With observed but diluted orbital amplitudes of $K=72$ and $37 \mathrm{~km} \mathrm{~s}^{-1}$, it was estimated that NGC 3603 and R 136 must contain 2-3 and 4- 5 WR stars, respectively. We now know from high-resolution images that the correct numbers are 3 and 4, respectively. But which WR stars have these orbits? As a first step to answer this in the case of NGC 3603, Moffat et al. (in preparation) analysed two years of HST-NICMOS focus-test $J$-band images (43 best-focus images being obtained) to deduce that it is star WR 43a that exhibits a double-eclipse light curve with amplitudes close to $0.1 \mathrm{mag}$ and $P=3.772$ days! Unfortunately, the only modest precision of the $H S T$-NICMOS photometry with $\sigma(J) \simeq 0.04$ mag failed to reveal any other clear periodic variable stars. A preliminary lightcurve fit to star WR 43a by E. Antokhina yields $i \simeq 70^{\circ}$ and $q \simeq 0.5-2$. Then with $K(\mathrm{WR} 43 \mathrm{a}) \simeq 3 K(4686) \simeq 216 \mathrm{~km} \mathrm{~s}^{-1}$, one finds a mass function of $2.5 \mathrm{M}_{\odot}$. Then assuming that the visible star in the spectrum of star WR $43 \mathrm{a}$ is the more 
massive component, one finds triplets of $\left[q, \mathrm{M}(\mathrm{WR} 43 \mathrm{a}) / \mathrm{M}_{\odot}, \mathrm{M}(2) / \mathrm{M}_{\odot}\right]=[1.0$, $12,12] ;[1.5,28,19] ;$ and $[2.0,81,27]$. While the last triplet seems reasonable, clearly one must await a detailed study using resolved spectroscopy.

\section{Winds, color-magnitude diagram, interstellar matter}

These are best probed using radio, IR and X-ray data. Radio and X-ray fluxes are often related via shocks.

\subsection{The XMEGA campaign}

During 2001, $50 \mathrm{ksec}$ of Chandra-ACIS-I $0.5-10 \mathrm{keV}$ X-ray images and four 12hour shifts of ATCA 3- and 6-cm radio images were obtained for NGC 3603 (Moffat et al. 2002; Mücke et al. 2002). Both are at $1^{\prime \prime}$ resolution, closely matched to other optical/IR data, which is a great advantage for comparisons.

\subsection{Summary of results}

Despite the opening paragraph in this section, radio and X-rays were found to be mostly uncorrelated, given their mostly thermal nature, probing gas at vastly different temperatures. Some exceptions do occur (see below).

Although the initial goal of the radio observations was to detect the O/WR winds, the only stellar-wind radio flux arises in the very core of NGC 3603 from dense subgroups of stars near objects WR 43a and WR 43b. This resolved radio emission had a total flux of $S_{\nu}=3.3 \pm 0.3 \mathrm{mJy}$ (at $3 \mathrm{~cm}$ ), which is expected for normal mass-loss rates from $\sim 10$ hot O/WNL stars. However, with $S_{\nu} \propto \nu^{\alpha}$ one finds $\alpha=-0.2 \pm 0.2$, so that some of the radio emission must be non-thermal, probably arising in wind-wind collisions (WWC).

In the whole Chandra-ACIS-I field, 384 point sources were detected at the $3-\sigma$ level. Forty-five of these coincide with known O/WR members of NGC 3603 (Melnick et al. 1989; Moffat 1983; Drissen et al. 1995). From these 45 stars, a mean trend of $L_{\mathrm{x}} \simeq 10^{-7} L_{\mathrm{bol}}$ is seen, with enormous scatter, \pm 1 dex on either side of this relation. Both of these findings are normal, although now extended to very hot, luminous stars. All the brightest known, early O-type stars and the three WN6h+abs stars were detected in $L_{\mathbf{x}}$, suggesting that the three WR stars behave more like luminous O-type stars than true, classical H-poor WR stars. The two late-O/early-B supergiants (including the SN 1987A-like star Sher 25, B1.5I) were not detected, probably because of their lower temperature, hence lower bolometric luminosity and slower wind velocity.

Outstanding X-ray emitting member stars include: $(i)$ the WR star WR 43c, which has an $L_{\mathrm{x}}$ like that of the Galactic record star WR 25 in the Carina Nebula. However, this star was not detected in the radio. Presumably, this is a result of a WWC in a medium-period WR+O binary; (ii) the two OB stars MTT 68 and MTT 71, located at projected separations of $\sim 3 \mathrm{pc}$ from the centre of NGC 3603. Recent spectra of these stars (Skalkowski et al., in preparation) show that they are very hot O-type stars like those found in the cluster core, one with emission lines. They suffer about $1.5 \mathrm{mag}$ more visual extinction than the core of NGC 3603, making them optically relatively faint. Their outstanding X-ray fluxes remain a mystery (but see $\S 5$ below); (iii) the bright WR star WR 43a (= A1, blended with O-type stars A2 and A3), which is the $3.772 \mathrm{~d}$ eclipsing binary. Hence this may well by excess X-rays arising in its colliding 
winds, although the orbital separation is rather close to allow X-rays to escape; and (iv) MTT11, which according to its spectrum is a $03-4 \mathrm{~V}+\mathrm{O} 4-5 \mathrm{~V}$ binary, so again its enhanced X-rays may be due to WWC.

Some of the detected X-ray point sources have no optical counterparts, yet their number increases (as for identified sources) towards the cluster centre. No significant variability was detected in any of the brightest X-ray sources, for which there are adequate counts, over time-scales of $0.5-50 \mathrm{ksec}$.

After removing recognized $\mathrm{X}$-ray point sources and extrapolating a gaussian fit into the confused central cluster core, a clear residual diffuse X-ray emission component was detected, reaching out to $r \simeq 4 \mathrm{pc}$, much like the extent of the PMS population. The total diffuse component is estimated to be $\sim 10^{34.3} \mathrm{erg} \mathrm{s}^{-1}$, which is $\sim 20 \%$ of the total observed X-ray flux from NGC 3603 . The total flux must therefore be dominated by detected point sources, contrary to the predictions of Cantó et al. (2000). The diffuse X-ray flux must arise from large numbers of merging/colliding hot-star winds and/or numerous faint, unresolved cluster sources. The diffuse spectrum leads to a temperature $k T \simeq 2-3 \mathrm{keV}$, which is hotter than the typical spectrum of the hot O-type stars $(\sim 0.9 \mathrm{keV})$.

The three proplyd-like objects discovered in NGC 3603 by Brandner et al. (2000) have been detected and resolved (size: several arcseconds) at 3 and $6 \mathrm{~cm}$. These are the most massive, luminous and distant objects of their kind detected so far. Spectral-index maps reveal non-thermal radio emission components arising in the tails of these 3 objects, compared to thermal emission from the heads facing the central hot sources. Compared to the over 100 proplyds encountered in the nearby Orion Nebula, with only one dominating source of ionization ( $\theta^{1}$ Ori C in the central Trapezium), the few NGC 3603 proplyd-likes are each 100 times more luminous, 100 times further from their central source, 100 times more massive and roughly 100 times larger in physical size.

\section{Exotica}

The central WR stars clearly stand out as being by far the most luminous stars in all of NGC 3603, making it tempting to label them 'Luminous Stragglers' (as opposed to the more familiar 'Blue Stragglers', since the main-sequence in NGC 3603 is nearly vertical for the most massive stars). Two possibilities come to mind to explain these exotic WR stars: $(i)$ due to mass segregation and binary formation particularly in the dense core, they may be products of stellar collisions, as is claimed for some of the most luminous stars in R 136 by Portegies Zwart et al. (1999). This is feasible, since the relaxation time is of the same order as the nuclear age of the stars; $(i i)$ or, we may be simply seeing the massive tail of the IMF. However, in this latter case, one wonders why we don't see the same massive tail among an equal number of massive stars outside the dense core. In support of scenario $(i)$, the two separated hot member stars MTT68 and MTT 71 might owe their strong X-ray fluxes to a recent, rapid ejection from the core via this stellar collision process. They are too young to have become massive X-ray binaries, although WWC in massive binary pairs is another possibility. Furthermore, Tenjes et al. (2001) have traced back the $5 \mathrm{M}_{\odot}$ extreme runaway star HIP 60350 (B4-5V) $\left(v_{\text {pec }}=-417 \mathrm{~km} \mathrm{~s}^{-1}\right)$ to NGC 3603 about 20 Myrs ago. This is too far back to be related to the current $1 \mathrm{Myr}$ cluster, but may be related to a previous burst of star formation from the same region. 
Ebisuzaki et al. (2001) predict that intermediate-mass black holes (IMBHs) may exist in young compact star clusters like R 136 (e.g., many are seen in M 82). Could this also be the case for the R 136-clone NGC 3603? In contrast to wellknown stellar and galactic-nuclei black holes of masses $\sim 10$ and $\sim 10^{6-9} \mathrm{M}_{\odot}$, respectively, so far unconfirmed IMBHs should have masses of $\sim 10^{2-6} \mathrm{M}_{\odot}$. The velocity field of stars near the core of NGC 3603 may be able to help decide in this case.

\section{Conclusions}

NGC 3603 is a somewhat younger, hotter, scaled-down version of typical starbursts found in other galaxies. With 'only' $L_{\mathbf{x}}$ (total unabsorbed, $\left.0.5-10 \mathrm{keV}\right) \simeq$ $10^{35} \mathrm{erg} \mathrm{s}^{-1}$, NGC 3603 pales compared to 30 Dor, with $L_{\mathbf{x}}$ (total unabsorbed, $0.1-2.4 \mathrm{keV}) \simeq 6 \times 10^{37} \mathrm{erg}^{-1}$, but with only $1-2 \%$ from massive stars, the rest from supernova remnants and massive $\mathrm{X}$-ray binaries, which do not yet exist in the co-eval NGC 3603 cluster. With $\mathrm{k} T \simeq 0.3 \mathrm{keV}$ for the average 30 Dor X-ray emission (as in most other starbursts), this is low compared to the $3 \mathrm{keV}$ from NGC 3603 . Thus 30 Dor is significantly more expanded and older on the whole.

\section{References}

Brandl, B., Brandner, W., Eisenhauer, F., Moffat, A.F.J., Palla, F., Zinnecker, H. 1999, A\&A (Letters) 352, L69

Brandl, B., Sams, B., Bertoldi, F., Eckart, A., Genzel, R., Drapatz, S., Hofmann, R., Löwe, M., Quirrenbach, A. 1996, ApJ 466, 254

Brandner, W., Grebel, E.K., Chu, Y.-H, Dottori, H., Brandl, B., Richling, S., Yorke, H.W., Points, S.D., Zinnecker, H. 2000, AJ 119, 292

Breysacher, J., Azzopardi, M., Testor, G. 1999, A\&AS 137, 117

Cantó, J., Raga, A.C., Rodríguez, L.F. 2000, ApJ 536, 896

Crowther, P.A, Dessart, L. 1998, MNRAS 296, 622

de Koter, A., Heap, S.R., Hubeny, I. 1998, ApJ 509, 879

Drissen, L., Moffat, A.F.J., Walborn, N.R., Shara, M.M. 1995, AJ 110, 2235

Drissen, L. 1999, in: K.A. van der Hucht, G. Koenigsberger \& P.R.J. Eenens (eds.), Wolf-Rayet Phenomena in Massive Stars and Starburst Galaxies, Proc. IAU Symp. No. 193 (San Francisco: ASP), p. 403

Ebisuzaki, T., Makino, J., Tsuru, T.G., Funato, Y., Portegies Zwart, S., Hut, P., McMillan, S., Matsushita, S., Matsumoto, H., Kawabe, R. 2001, ApJ (Letters) 562, L19

Grebel, E.K., Harbeck, D., Brandner, W., Stolte, A., Odenkirchen, M., Drissen, L., Moffat, A.F.J., Chu, Y.-H. 2003, in preparation

van der Hucht, K.A. 2001, New Astron. Reviews 45, 135

Hunter, D.A., Shaya, E.J., Holtzman, J.A., Light, R.M., O'Neil, E.J., Lynds, R. 1995, ApJ 448, 179

Hunter, D.A., O'Neil, E.J., Lynds, R., Shaya, E.J., Groth, E.J., Holtzman, J.A. 1996, ApJ (Letters) 459, L27

Massey, P.M., Penny, L.R., Vukovich, J. 2002, ApJ 565, 982

Melnick, J., Tapia, M., Terlevich, R. 1989, A\&A 213, 89

Moffat, A.F.J. 1983, A\&A 124, 273

Moffat, A.F.J., Seggewiss, W. 1983, A\&A 125, 83

Moffat, A.F.J., Niemela, V.S. 1984, ApJ 284, 631 
Moffat, A.F.J., Seggewiss, W., Shara, M.M. 1985, ApJ 295, 109

Moffat, A.F.J., Drissen, L., Shara, M.M. 1994, ApJ 436, 183

Moffat, A.F.J., Corcoran, M.F., Stevens, I.R., Skalkowski, G., Marchenko, S.V., Mücke, A., Ptak, A., Koribalski, B.S., Brenneman, L., Mushotzky, R., Pittard, J.M., Pollock, A.M.T., Brandner, W. 2002, ApJ 573, 191

Mücke, A., Koribalski, B.S., Moffat, A.F.J., Corcoran, M.F., Stevens, I.R. 2002, ApJ 571, 366

Nürnberger, D.E.A., Petr-Gotzens, M.G. 2002, A\&A 382, 537

Portegies Zwart, S.F., Makino, J., McMillan, S.L.W., Hut, P. 1999, A\&A 348, 117

Schmutz, W., Drissen, L. 1999, RMxAA-SC 8, 41

Selman, F.J., Melnick, J., Bosch, G., Terlevich, R.J. 1999, A\&A 347, 532

Smith, L.F., Shara, M.M., Moffat, A.F.J. 1996, MNRAS 281, 163

Tenjes, P., Einasto, J., Maitzen, H.M., Zinnecker, H. 2001, A\&A 369, 530

\section{Discussion}

KUDRITZKI: I do not think that the scatter in $L_{\mathrm{x}} / L_{\mathrm{bol}}$, which you find, is unusually large. The old Einstein/ROSAT sample of galactic O-type stars shows a similar scatter.

Moffat: Agreed, but it does show that for an extremely young, dense cluster, the same conditions hold, going up to the earliest hottest O-type stars and even very luminous WNLh stars.

MAEDER: How well do correspond the ages for NGC 3603 that you determine from the upper MS turnoff and from the pre-MS evolution of low mass stars?

MOFFAT: As time passes, it seems that these two are converging to a value close to $\sim 1$ Myr. Taken at face value, though, there is still the possibility that the pre-MS population is slightly younger on average, compared to the post-MS population.

ZINNECKER: $(i)$ A note of caution: any stellar ages below 1 Myr derived from current pre-MS models are bound to be unreliable. So the jury is still out whether the massive stars and the lower mass pre-MS stars in NGC 3603 are co-eval, or whether one population is somewhat younger/older than the other (cf. Eisenhauer et al. 1998, ApJ 498, 278 ), i.e., which came first. (ii) I also have a quick question: When you say that 30 Dor is 10 times NGC 3603, what do you really mean? Do you mean 10 times the number of massive stars?

MOFFAT: $(i)$ Right, but nevertheless, the ages from post- and pre-MS have been converging recently in NGC 3603 to a more or less co-eval value of $\sim 1 \mathrm{Myr}$. (ii) $30 \mathrm{Dor}$ contains an order-of-magnitude more OB/WR stars than NGC 3603. Being larger by $\sim 10$ times, this is not surprising.

MAÍZ-APELLÁNIZ: Would you agree that the difference in size between R 136 and NGC 3603 on the one hand and NGC 1705-IA and NGC 1569-B on the other hand $(\sim 1 \mathrm{pc} v s . \sim 3 \mathrm{pc})$ is due to the mass loss by stellar winds and SN explosions, given that the first two objects have not gone through the most violent mass loss phase?

Moffat: Sounds quite reasonable, as R.A.W. Elson et al. (ApJ 336, 734) showed, although there they also included stellar winds as well as SNe in their models. 